\title{
A Face Detector Based On Flexible Matching
}

\author{
Chen Guang ${ }^{1, a}$ \\ ${ }^{1}$ Department of Computer and Electronics Information Engineering, \\ Hubei University, \\ Wuhan, China. \\ abookslave@163.com
}

Keywords: Face detection, Hausdorff distance.

Abstract. Face detector is the first stage for some biometric applications, like face recognition or advanced human computer interaction, which usually needs the face detection step to be efficient and robust enough to achieve better results. In this paper Hausdorff distance is used to search interested region, which is more similar to a face. A further verification step is taken to detect some false positives. Experiments show that the proposed face detector can perform accurate face detection even with complex background.

\section{Introduction}

Face detection is the first step in automated face recognition. Many biometric applications rely on a reliable face detector. Results of these applications will not be robust enough if face detection is not accurate [1][2][3]. Face detection can be performed based on several different cues: Skin color, motion, shape, and facial appearance. Prior knowledge about them can be achieved by learning from training data.

In this paper, we propose an improved face detection system based on Hausdoff distance. we introduce the Hausdorff metric we use, and how it is computed more efficiently. Then, we describe how our detection system works, and some special measures be taken to improve the system's detection rate and robustness. Finally, some experimental results are shown, and some conclusions are referenced .

\section{Hausdorff Distance}

Hausdorff distance is a metric for comparing points of two patterns. It is used in pattern recognition to determine the degree of resemblance between two patterns. The Hausdorff distance between two patterns, $\mathrm{C}$ and $\mathrm{D}$, is defined as[8]:

$$
H(C, D)=A \vee G h(C, D), h(D, C))
$$

$$
h(C, D)=\frac{1}{C \mid} \sum_{c \in C} M I N\|C-d\|_{d \in D}
$$

$$
h(D, C)=\frac{1}{|D|} \sum_{d \in D} M I N\|d-C\|_{C \in C}
$$

Let $\mathrm{C}$ be a representation of the image to be searched, and $\mathrm{D}$ a model presentation of the pattern we want to search. Let $T=\left(t_{x}, t_{y}, s_{x}, s_{y}\right)$ be the representation of space transformation, where $t_{x}$ and $t_{y}$ represent translation, and $s_{x}$ and $s_{y}$ scale. In order to find the object in C, we need to find some transformed model $T(D)$ to make: 


\section{Detection Theory}

Figure 1 shows the complete face detection process, which consist of a preprocessing step, a detection step and a post-processing step. The preprocessing step transforms the input image into a binary edge image. The detection process utilize the Hausdorff distance to search the object similar to the face template, some measures are taken to exclude some false positive detections at the third step.

Preprocessing operations are usually performed to normalize the image pattern in its size, pixel value distribution and light conditions. First, we resize the input image below 500x500 pixels to decrease the computation time, then histogram normalization follows, finally the Sobel operator is used to extract edges from the input image.

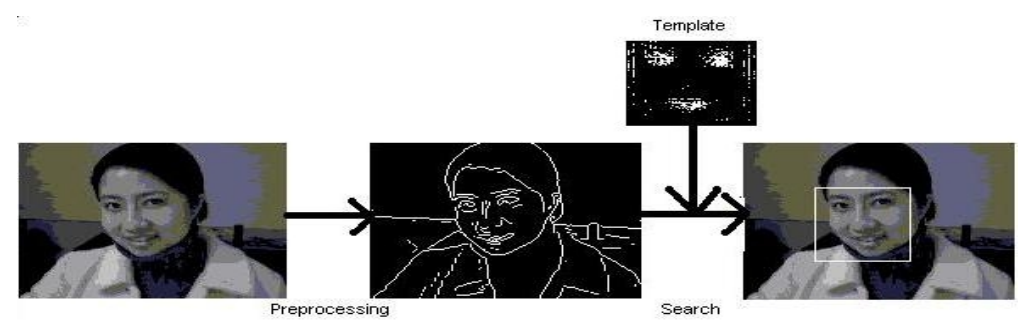

Figure 1. The face detection system

In order to search the binary image we have got at previous step, we need to create a face template. We extract the face template from the images downloaded during a random crawl of the WWW. The Faces are only roughly aligned, which are cropped and scaled to $100 \times 100$ pixels. The face database consists of 1,034 faces .We generate the face template using the average edge map of the 1,034 faces, which is shown in Figure 2:

The whole search process is summarized as follows:

- The template is scanned across the image at multiple scales and locations defined by $T$. At each search position, we get the values of $H(C, T(D)), N_{s}, N_{t}$, using the method in section 2 .

- When $H(C, T(D))<\mathrm{e}, N s / C_{s}<l_{s}$, and $N_{t} / C_{t}<l_{t}$, we find a possible object pattern. $C_{s}$ represents the number of $P_{s}, C_{t}$ represents the number of $P_{t}$, and $e, l_{s}, l_{t}$ are the thresholds.

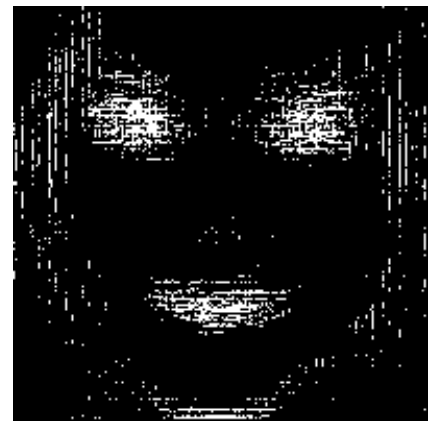

Figure 2. Face template

Usually Hausdorff distance values are lower for smaller template size, which means that if the face in the searched image is large, correct position and scale may have a larger distance than those positions with small scales. We adjust the threshold e according to different scale[8]. But it is not wise enough to exclude most false positives while keeping a high face detection rate. Furthermore, we adopt a very simple method to solve the problem: 
- Based on the search results in step 2, we select all positions with local minimum Hausdorff distance for each scale.

- Compute the smallest distance $H_{m i n}$, from all local minimums, then exclude those positions which satisfy $H_{l o c}>r H_{m i n}$, where $H_{l o c}$ represents the local minimum Hausdorff distance for each selected position, $\mathrm{r}$ is the ratio just above 1 , which is also determined by validation set.

The method is based on the observation that at the same scale, the Hausdorff distance of the object pattern is usually smaller than other patterns, Figure 3 gives an example of the observation.

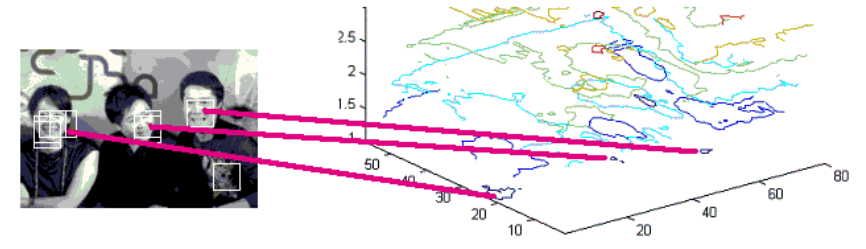

Figure 3. Hausdorff distance map at some scale

Through the method, most false positives can be eliminated. However, a face may be detected several times at multiple close scales. A heuristic method can be adopted to solve the problem. A detect is further confirmed if the number of multiple detections is large enough, and multiple detections at close scales are merged into a consistent detect. Figure 4 gives an illustration:

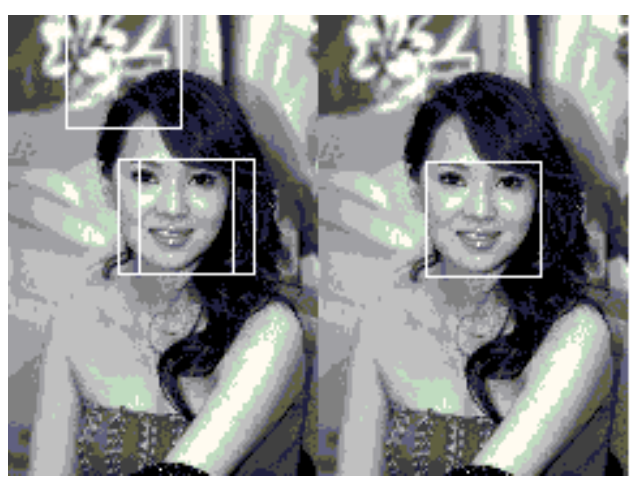

Figure 4. Merge multiple detections

\section{Experimental Results}

The test data set used in experiments was the MIT+CMU set[5], which consists of 130 images with 507 frontal faces. The validation set used to determined the thresholds is the FERET set[2]. By experiment, we select $1_{t}=0.005,1_{s}=0.055, \mathrm{r}=1.11$, and $\mathrm{e}=0.75 \sqrt{\left(s_{x}+s_{y}\right) / 2}$ [8] $, s_{x}, s_{y}$ are scale coefficients.

Table. 1 give the detection result for MIT+CMU set

TABLE I. TEST RESULT FOR MIT+CMU DATE SET

\begin{tabular}{cccc}
\hline \multirow{2}{*}{ Detector } & \multicolumn{3}{c}{ False detects } \\
\cline { 2 - 4 } & 25 & 97 & 167 \\
\hline The proposed & 80.4 & 94.7 & 96.6 \\
\hline
\end{tabular}

Figure.5. give some detection examples. 


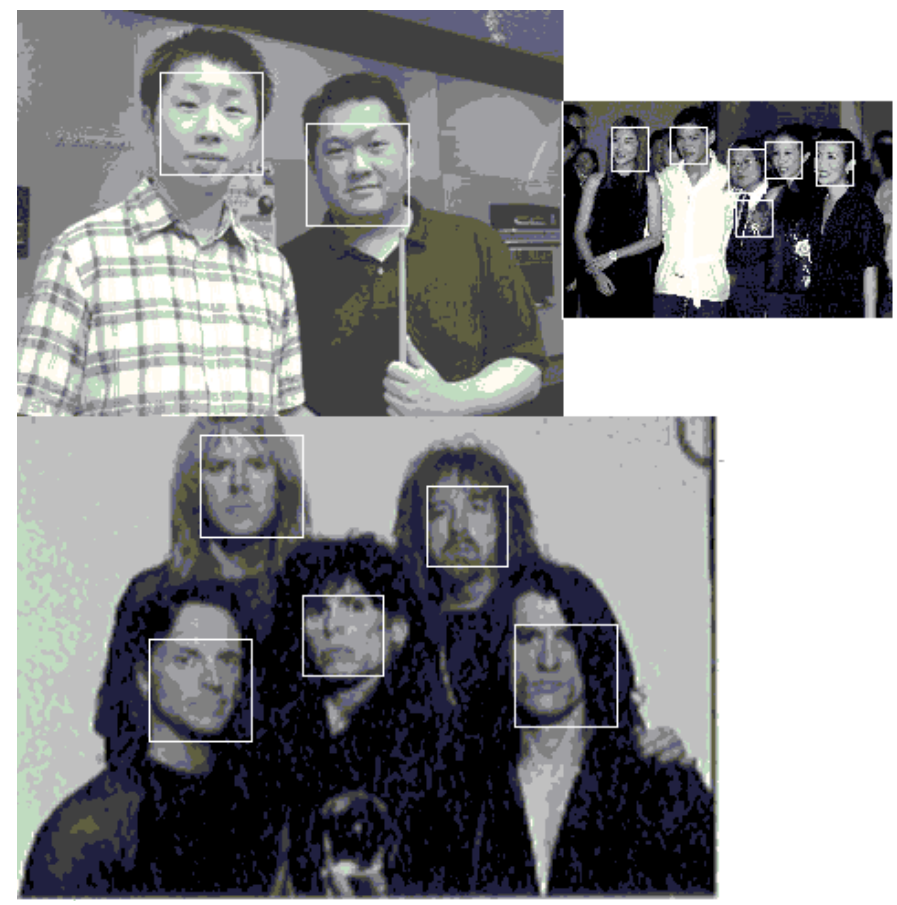

Figure 5. Some detection result

\section{Conclusions}

We have proposed a robust face detection system based on Hausdorff distance. The detector is flexible enough to detect faces in images with complex background, and some not frontal faces. However, the thresholds can only be selected by experiments through validation set, which indicates that we should define an optimization framework in future work.

\section{References}

[1] VIOLA Paul, "Robust real-time face detection, International Journal of Computer Vision", 2004, vol. 57 , pp. 137-154.

[2] P. J. PHILLIPS, “The FERET evaluation methodology for face recognition algorithms", Image and Vision Computing, 1998, vol. 16, pp. 295-306.

[3] CHELLAPPA Rama, "Human and machine recognition of faces, a survey", Proceedings of the IEEE, 1995, vol. 83, pp. 705-740.

[4] S. Z. LI, "Face recognition using the nearest feature line method", IEEE Transactions on Neural Networks, 1999, vol. 25, pp. 439-443.

[5] H . ROWLY, "Neural network-based face detection", IEEE Transaction on PAMI, 1998, vol. 20, pp. 23-28.

[6] G. YANG, "Human face detection in complex background", Pattern Recognition, 1994, vol. 27, pp. $53-63$.

[7] YANG Minghsuan, "Detecting faces in images : A survey",IEEE Trans on Pattern Analysis and Machine Intelligence,2002, vol. 24 , pp. 34-58.

[8] Huttenlocher, "Comparing Images using the Hausdorff Distance", IEEE Transactions on Pattern Analysis and Machine Intelligence,1993, vol 14, no.9, pp. 850-853. 